\title{
A influência e a percepção das cinco forças competitivas de Michael Porter: um estudo no setor empresarial de Sobral - CE
}

\author{
Evandro Souza $^{\mathrm{a}}$, Sefisa Quixadá Bezerra ${ }^{\mathrm{b}}$, Levi Leonido ${ }^{c}$, Luís André Aragão Frota ${ }^{\mathrm{d}}$, \\ Elsa Morgado ${ }^{\mathrm{e}}$ \\ ${ }^{a}$ Universidade Estatual do Vale do Acaraú, Sobral, Brasil, evandrosales7@ @otmail.com, \\ ${ }^{\mathrm{b}}$ Universidade Estatual do Vale do Acaraú, Sobral, Brasil, sefisaquixada@ gmail.com ${ }_{2}^{\mathrm{c}}$ Universidade \\ de Trás-os-Montes e Alto Douro, Vila Real, Portugal, Universidade Católica Portuguesa (CITAR), \\ Porto, Portugal, levileon@utad.pt, ${ }^{\mathrm{d}}$ Instituto Federal do Piau, Cocal, Brasil, \\ luisandrearagao@yahoo.com.br, ${ }^{\mathrm{e} U n i v e r s i d a d e ~ C a t o ́ l i c a ~ P o r t u g u e s a ~-~ C e n t r o ~ d e ~ E s t u d o s ~ F i l o s o ́ f i c o s ~}$ \\ e Humanísticos - Braga, UTAD-Vila Real, IPB-Bragança, Portugal, levielsa@utad.pt
}

\section{Resumo}

Este trabalho aborda o estudo das Cinco Forças Competitivas de Porter no setor empresarial (panificação) da cidade de Sobral - CE, com o objetivo de analisar a percepção dos empresários com relação à atuação dessas forças no setor local. Como referencial teórico, o estudo inicia com uma abordagem a respeito das Cinco Forças Competitivas de Porter como instrumento para o planejamento estratégico e uma caracterização de cada uma destas forças. A parte dedicada ao estudo empírico traz os resultados de uma pesquisa de caráter quantitativo realizada com os empresários das padarias utilizando um instrumento estatístico que possibilita uma análise segura dos dados (Teste Qui-Quadrado), os quais revelam as percepções dos mesmos acerca da intensidade das Forças de Porter dentro do setor na cidade de Sobral. Com as informações obtidas, foi possível concluir que apenas uma delas possui forte atuação no setor. As demais, na visão dos empresários, apresentam uma atuação tímida. Essas forças com atuação tímida e pouca agressiva, atualmente podem não ser vistas como ameaças, mas a longo prazo elas podem reestruturar-se e atingirem fortemente o desempenho do setor.

Palavras-chave: Forças competitivas, empresas, estratégias. 


\begin{abstract}
This work deals with the study of Porter's Five Competitive Forces in the business sector (breadmaking) of the city of Sobral - CE, with the objective of analyzing the entrepreneurs' perception regarding the performance of these forces in the local sector. As a theoretical reference, the study begins with an approach regarding the Five Porter Competitive Forces as an instrument for strategic planning and a characterization of each of these forces. The part devoted to the empirical study brings the results of a quantitative research carried out with the entrepreneurs of the bakeries using a statistical instrument that allows a safe analysis of the data (Chi-Square Test), which reveal their perceptions about the intensity of the Forces of Porter within the sector in the city of Sobral. With the information obtained, it was possible to conclude that only one of them has strong performance in the sector. The others, in the view of the entrepreneurs, present a timid performance. These timid, low-aggressive forces today may not be seen as threats, but in the long run they can restructure and strongly achieve industry performance.
\end{abstract}

Keywords: Competitive forces, interprise, strategies.

\title{
1. Introdução
}

Nos últimos anos, diversos estudos têm sido desenvolvidos com o objetivo de compreender o que leva determinados setores a obterem melhor desempenho no mercado, maior participação, reconhecimento e, consequentemente, maior lucratividade, e o que leva outros a falharem ou obter sucesso na adoção de um posicionamento estratégico. A Associação Brasileira da Indústria de Panificação e Confeitaria (ABIP), importante referencia brasileira de panificação, revela que o setor apresentou um índice de crescimento de 8,02\% em 2014, o que representa um faturamento de $\mathrm{R} \$ 82,5$ bilhões (ABIP, 2015). O estudo demonstra que este é o segundo ano consecutivo que o setor tem um crescimento inferior a $10 \%$, apresentando a menor taxa dos últimos oito anos, contudo a taxa de crescimento continua positiva. Entretanto, como aponta o estudo, mesmo passando por esse momento de desaceleração, as panificadoras representam o segundo setor que mais cresce no país, ficando a sua frente apenas os supermercados. O Programa de Apoio à Panificação (Propan) mostrou que 66\% dos brasileiros consomem pão no café da manhã e $98 \%$ da população são consumidores de produtos panificados. Essas informações constatam a forte presença das panificadoras no cotidiano das famílias residentes neste país, demonstrando a importância desse setor para a economia (SEBRAE, 2009; ABIP, 2015). Diante destas 
informações, desenvolver estudos que constatem essa realidade e identifiquem as ações gerenciais que criam e mantêm um posicionamento estratégico que tornam o setor sustentável, é contribuir para o fortalecimento da economia do país e do setor. Diante desta contextualização prévia torna-se fundamental responder às seguintes qustões: De que forma os gestores percebem o impacto das Cinco Forças Competitivas do modelo de Porter no desempenho estrutural das panificadoras de Sobral? As estratégias genéricas de Porter são adotadas pelas panificadoras como ferramenta para posicionar-se estrategicamente e obter um diferencial competitivo? E, por fim, Qual (is) força(s) é (são) percebida(s) com mais influência pelos gestores? Este trabalho tem como objetivo principal a análise e a percepção dos empresários com relação a influência de cada uma das Cinco Forças Competitivas de Porter no setor na cidade de Sobral-CE-Brasil.

\section{Enquadramento}

Michael Porter é um dos autores pioneiros no estudo da competitividade global e industrial. Os seus trabalhos têm sido recorrentemente utilizados nas ações de planejamento estratégico, que procuram identificar a competitividade das empresas a partir da análise de fatores ambientais. Este é intitulado de artesão da estratégia empresarial (Barney, 1997; Bignetti \& Paiva, 2001; Bertero, Vasconcelos \& Binder, 2003; Ramoz-Rodrigues \& Ruiz Navarro, 2004; Mercês, 2009). O modelo das Cinco Forças de Porter pode ser compreendido como uma ferramenta que auxilia a definição de estratégia da empresa e leva em consideração tanto o ambiente externo como o interno, começando por ampliar o conceito de concorrência, sendo essa caracterizada por disputas por parcelas de mercado entre as empresas de um mesmo ramo de negócio que produzem produto e/ou serviços iguais ou semelhantes que satisfazem as mesmas necessidades dos clientes atuais ou futuros (Porter, 1990). Para o autor, a concorrência deve ser vista considerando-se as seguintes forças: Ameaça de novos concorrentes; Rivalidade em relação aos concorrentes existentes; Ameaça de produtos e serviços substitutos; Poder de compra dos clientes; e Poder de negociação dos fornecedores. O estudo de Porter (1990) assenta na seguinte premissa: quanto maior a força, menor a rentabilidade estrutural de setor e, consequentemente, da empresa. Assim, se um setor é caracterizado pela intensa concorrência; fornecedores e compradores com alto poder de troca, os quais conseguem impor com facilidade as suas condições; com forte concentração de produtos substitutos e no qual muito fácil entrar, inexistindo muitas barreiras de entrada, em longo prazo, dificilmente será um setor rentável (Fernandes \& Berton, 2005). Ainda segundo esses autores, a presença dessas forças num setor, somada à intensidade de cada uma delas, estabelece o potencial de desempenho de um mercado. A análise isolada de cada uma dessas ameaças possibilita a identificação dos 
A influência e a percepção das cinco forças competitivas de Michael Porter: um estudo no setor empresarial de Sobral - CE

elementos que compõem a estrutura de um setor, diagnosticando-o. Isso significa que identificando os fatores que favorecem ou depreciam um setor, é possível transformar as ameaças em oportunidades (Porter, 1990).

\section{Metodologia}

O trabalho insere-se numa pesquisa de campo de natureza Quali-quantitativa e exploratória (Gil, 2008). Os instrumentos utilizados para a coleta de dados: observação atenta aos detalhes; aplicação de entrevistas semi - estruturadas a 11 proprietários do setor, e um questionário fechado aplicado a 27 gerentes e/ou proprietários de padarias da cidade de Sobral-CE - Brasil.

As entrevistas semi-estruturadas foram norteadas pelos tópicos relacionados às forças competitivas, permitindo que os entrevistados manifestassem livremente a sua opinião, (Alencar, 2009). A escolha do questionário deveu-se ao fato de ser necessária a obtenção das características da população estudada para perceber a relação entre as variáveis, confirmando, assim, nesse estudo a existência da pesquisa descritiva como um importante instrumento na aplicação e no sucesso da pesquisa quantitativa. O questionário contou com questões referentes à atuação das Forças Competitivas de Porter no setor que se caracterizaram como variáveis nominais do tipo Sim e Não e que serviram para acumular dados para uma análise mais aprofundada das forças, especialmente na identificação da relação existentes entre elas. Os dados qualitativos foram analisados através de "análise de conteúdo" que, segundo Vergara (2010, p.7) é uma técnica utilizada para se "analisar o que está sendo dito a respeito de determinado tema". Esta técnica permitiu a construção de explicações com base nas interpretações de conteúdos categorizados previamente por meio da comparação. Os dados foram agrupados em cinco categorias analíticas que se referiam a cada uma das Cinco Forças Competitivas de Porter e permitiram o aprofundamento do que ficou obscuro na aplicação do instrumento quantitativo. Os dados quantitativos foram organizados em categorias analíticas de acordo com a relação que existia com as forças apontadas no estudo. A partir de então foi criada uma hipótese nula e uma hipótese alternativa para cada força analisada. Sendo denominadas H0 e H1 respectivamente. Nos métodos estatísticos, o uso de hipóteses trazem afirmações a respeito da população. A escolha por uma ou outra hipótese está condicionada ao que é chamado de valor-p trazido pelos dados e ao nível de significância pré-estabelecido pelo estudo (Agresti \& Finlay, 2012). Segundo Agresti e Finlay (2012), se o valor-p for menor que determinado nível de significância (Sig.), passa-se então a rejeitar a hipótese nula e consequentemente aceita hipótese alternativa, em caso contrário, o inverso ocorre. Ainda segundo os autores, o nível de significância padrão deve ser 0,05 para a tomada de decisão. Ou seja, para $p \geq 0,05$ 
aceita-se a hipótese nula (H0) e para $p \leq 0,05$ aceita-se a hipótese alternativa (H1). Neste estudo, para chegarmos ao valor - $p$ foi usado o programa SPSS. Os dados foram analizados pelo método Qui-Quadrado. Este método é caracterizado como não paramétrico que analisa a hipótese nula de não existência de discrepância entre as frequências observadas nas variáveis em estudo, ou seja, a não existência de associação significativa entre as variáveis (Bruni, 2012; Agresti \& Finlay, 2012). A escolha deste método está associada às características da amostra, pois segundo Agresti e Finlay (2012) quando as amostras são numericamente pequenas $(\mathrm{n} \leq 30)$ e não seja possível verificar a normalidade dos dados do universo, existe a possibilidade de as mesmas não representarem a população, impossibilitando a construção de suposições. No caso desta pesquisa, para cada força de Porter analisada (categorias) foi elaborado uma tabela com os resultados dos questionários e aplicado um teste Qui-Quadrado que buscou verificar se existia alguma relação entre as respostas encontradas (variáveis). O teste obedece ao seguinte padrão de hipóteses:

Tabela 1. Relação das hipóteses com níveis de significância

\begin{tabular}{|l|l|l|}
\hline H0: São independentes & $\begin{array}{l}\text { Não existe associação significativa entre as } \\
\text { variáveis. }\end{array}$ & $\mathrm{p}>0,05$ \\
\hline H1: Não são independentes & Existe associação significativa entre as variáveis. & $\mathrm{p}<0,05$ \\
\hline
\end{tabular}

Fonte: Agresti e Finlay (2012)

Caso o valor-p seja maior que o nível de significância $(0,05)$ significa que as variáveis são independentes, portanto conclui-se que "não existe associação entre as variáveis" levando a aceitar a hipótese H0. Vale ressaltar que, ao adotar o valor do nível de significância igual a 0,05, podemos afirmar com $95 \%$ de certeza que a escolha da hipótese está correta, ou seja, se esse teste for refeito 100 vezes, 95 delas dará o mesmo resultado (Agresti \& Finlay, 2012).

\section{Resultados/Conclusões}

Ameaça de novos entrantes: A grande maioria dos entrevistados $(92,6 \%)$ afirmou que existem barreiras para quem está querendo entrar no setor de panificação em Sobral, fato percebido tanto por quem é novo no mercado quanto por quem já atua há mais tempo. Para analisar essa força através do teste Qui-Quadrado, consideraram-se "novos entrantes" aqueles estabelecimentos que tinham até 02 anos de existência no mercado e estabelecimentos "velhos" os que tinham acima de 02 anos de existência. Com a aplicação 
do teste Qui-Quadrado, o mesmo apresentou um nível de significância igual a 0,512 significando a aceitação da hipótese H0. Isso mostra que os entrevistados não percebem uma relação entre as perguntas. Ou seja, independentemente do tempo de atuação no mercado, tanto os novos entrantes quanto os mais antigos percebem a existência de barreiras. O estudo demonstrou que dentre as "barreiras" apontadas, a maior e de mais representatividade tanto para os novos entrantes como pelos mais antigos é a "escassez de mão de obra qualificada" (51,9\%). A explicação para esse fato é que em virtude dessa falta de profissionais qualificados, os gestores alegam que perdem competitividade, pois, necessitam treinar os funcionários recém-contratados, o que gera maiores custos e um período de tempo maior para que eles se adaptem à cultura interna da panificadora. Outra dificuldade, a ser superada pelos novos entrantes, é a "Legislação municipal" (14,8\%). Para os gestores interrogados, o município impõe uma série de exigências legais que retardam e acabam desestimulando o empreendedor a prosseguir com seu projeto de negócio. Com a mesma relevância percentual da anterior (14,8\%), a "necessidade de capital" também é um fator que dificulta a inserção no setor. Para os entrevistados, abrir uma padaria em Sobral ${ }^{1}$ exige um investimento financeiro alto, para além do investimento na estrutura física

Ameaça de produtos ou bens substitutos: Para analisar a percepção a respeito desta força foi feito um cruzamento entre as perguntas "existem produtos substitutos?" e "O seu produto é vulnerável a esses produtos substitutos?”. Do resultado foi aplicado o teste Qui-Quadrado que verificou a hipótese de independência ou associação entre essas perguntas. A hipótese H0 prevê a existência de independência entre as variáveis, ou seja, a não existência de relação entre as mesmas. E a hipótese $\mathrm{H} 1$ prevê o contrário: a não existência de independência entre as variáveis, logo, a existência de relação entre as mesmas. $\mathrm{O}$ cruzamento dessas perguntas obteve um nível de significância igual a 0,302. Assim é possível aceitar a hipótese H0 de que não há uma relação significativa entre as variáveis, ou seja, segundo a percepção do empresário o fato de existir um produto substituto no mercado não significa que o mesmo irá influenciar na sua venda. Porter (1999) esclarece que os produtos substitutos impõem um teto nos preços de uma empresa, podendo diminuir seus rendimentos. Atentando-se para os números, podemos justificar a opinião dos que relataram que são afetados pelos produtos substitutos, 29,6\% deles apenas, em virtude da existência de estabelecimentos que ofertam produtos iguais ou semelhantes aos das panificadoras..

Poder de negociação dos clientes: Para esta outra força foram cruzadas as perguntas "Seus produtos são iguais aos do concorrentes" e "Caso você aumente o preço do seu produto, o cliente deixa de comprá-lo?". Do resultado do teste Qui-Quadrado resultou um nível de significância igual a 0,001 . Desta forma rejeita-se a hipótese H0 e aceita-se a hipótese H1 de que existe uma relação entre as perguntas, mostrando que um pouco mais da metade dos

\footnotetext{
${ }^{1}$ IBGE (2012); IPCE (2012).
} 
entrevistados acreditam que o cliente pode deixar de comprar os produtos caso o preço dos mesmos venha a aumentar, significando que o poder de negociação do cliente, embora com baixa intensidade, é percebido pelos empresários do setor. O que explica a leve atuação dessa força no setor de panificação de Sobral é a alta concentração de padarias na cidade. Porter (1999) explica que, quando um setor é composto por inúmeras empresas que não apresentam grandes diferenciais competitivos, ou seja, seus produtos possuem atributos semelhantes aos do concorrente, os gestores possuem baixo grau de liberdade para impor aumentos de preços, exigir prazos ou recusar-se a dar descontos.

Poder de negociação dos fornecedores: A respeito desta outra força, teve-se em consideração as perguntas "Há facilidade na troca de fornecedores?" e "Há rigidez na alteração do preço do seu fornecedor?”. O resultado do teste analisou as hipóteses H0, que indica a não relação entre as variáveis por serem independentes, e a H1 que indica o contrário. O teste Qui-Quadrado apresentou um nível de significância igual a 0,040 mostrando a não aceitação da hipótese H0. Isso significa que os entrevistados percebem uma relação entre as perguntas, ou seja, para eles o fato de ter muitos fornecedores interfere na diminuição do poder de troca/negociação dos mesmos. Percebe-se que, se por um lado, o setor deve-se preocupar com o poder de troca/negociação dos compradores, a mesma atenção também deve ser dada ao poder de troca/negociação dos fornecedores, afinal essas forças ocupam as duas pontas da cadeia produtiva de um setor (Porter, 1999). A força poder de troca/negociação dos fornecedores possui baixa intensidade no setor, o que demonstra um baixo grau de dependência por parte dos atuais empresários na relação com seus fornecedores. Fernandes e Berton (2005), que aprofundaram o estudo de Porter no que refere à análise competitiva de um setor, esclarecem que um grupo fornecedor é poderoso quando um setor é dominado por poucos fornecedores, os quais impõem com facilidade suas políticas de preços, prazos, qualidade, dentre outros atributos. É possível, segundo os empresários, trocar de fornecedores sempre que estes estabelecem políticas desfavoráveis ou quando não cumprem os critérios das políticas atuais, tais como prazos, garantias, qualidade. Os empresários ainda relatam que existem disputas entre os próprios fornecedores, cada um tentando obter uma parcela maior de clientes, o que acaba por reduzir-lhes ainda mais seu poder de troca/negociação.

Rivalidade entre os concorrentes: A respeito desta outra força, foi levado em consideração as perguntas "Há muitos concorrentes no mercado de panificação?" e "Existe respeito entre os concorrentes?". A hipótese H0 levantada alega a inexistência de dependência entre as variáveis, mostrando que não estão relacionadas e a hipótese H1 alega o contrário. Para esta força, o teste Qui-Quadrado apresentou um nível de significância igual a 0,434 significando a aceitação da hipótese H0. Isso mostra que os entrevistados não percebem uma relação entre as perguntas, ou seja, para eles o fato de ter muitos concorrentes não 
A influência e a percepção das cinco forças competitivas de Michael Porter: um estudo no setor empresarial de Sobral - CE

interfere no respeito que existe entre os competidores. Segundo Porter (1999) a rivalidade tende a ser maior quando o setor encontra-se em recessão ou em baixo crescimento.

Porém, não basta conhecer e identificar a atuação de cada uma dessas Forças dentro do setor se esse conhecimento não for capaz de despertar o interesse dos empresários em desenvolver estratégias que possam melhorar o desempenho estrutural do setor. Foi com esse propósito que Porter (2008) desenvolveu as chamadas estratégias genéricas, sendo elas: a estratégia na Liderança do custo total; Diferenciação e Enfoque as quais são vistas como instrumentos capazes de responder às fragilidades diagnosticadas no estudo do setor, transformando-as em oportunidades que gerem um diferencial competitivo para o mercado. Pela análise dos resultados, foi possível concluir que apenas uma delas possui forte atuação no setor. As demais, na visão dos empresários, apresentam uma atuação tímida. Recorrendo ao raciocínio desenvolvido por Porter de que quanto maior a presença ou atuação das forças mais vulnerável torna-se o setor diante da ação da concorrência, podemos constatar que o setor de panificação não é vulnerável à forte pressão dos concorrentes. Isso significa que elas não impactam o desempenho estrutural do setor. Contudo, estas forças com atuação tímida e pouca agressiva, atualmente podem não ser vistas como ameaças, mas a longo prazo elas podem reestruturar-se e atingir fortemente o desempenho do setor.

\section{Referências}

ABIP (2015). Performance do setor de panificação e confeitaria brasileiro em 2015. Disponível em: http://www.abip.org.br/site/indicadores-2014

Agresti, A., \& Finlay, B. (2012). Métodos estatísticos para ciências sociais. São Paulo: Artmed.

Alencar, E. (2009). Metodologia de Pesquisa. Lavras: UFLA.

Barney, J. B. (1997). Gaining and Sustaining Competitive Advantage. New Jersey: Prentice Hall.

Bertero, C. O., Vasconcelos, F. C., \& Binder, M. P. (2003). Estratégia empresarial: a produção científica brasileira entre 1991 e 2002. Revista de Administração de Empresas, 43(4), 48-62.

Bignetti, L. P., \& Paiva, E. L. (2001). Estudo das Citações de Autores de Estratégia na Produção Acadêmica Brasileira. Encontro da ANPAD, 2001, Campinas, Brasil.

Bruni, A. L. (2012). SPSS Guia Prático para Pesquisadores. São Paulo: Atlas.

Fernandes, B. H. R. \& Berton, L. H. (2005). Administração Estratégica: da competência empreendedora à avaliação de desempenho. São Paulo: Saraiva.

Gil, A.C (2008). Como elaborar projetos de pesquisa. São Paulo: Atlas.

IBGE (2012). População Estimada de Sobral. Disponível em: www.ibge.gov.br 
IPECE (2012). Perfil básico municipal de Sobral. Disponível em: http://www.ipece.gov.br

Mercês, M. V. S. (2009). Posicionamento estratégico das empresas da construção civil em Pernambuco/Brasil, nos anos 2007 e 2008, diante da volta dos financiamentos bancários de longo prazo e do aumento da concorrência de grandes empresas. (Dissertação de Mestrado em Gestão). ISCTE - Instituto Universitário de Lisboa, Lisboa.

Porter, M. (1999). Competição = On competition: estratégias competitivas essenciais. Rio de Janeiro: Elsevier.

Porter, M. (2008). As cinco forças competitivas que moldam a Estratégia. Harvard Business Review. 86(1), 55-69.

Ramos-Rodriguez, A. R., \& Ruiz-Navarro, J. (2004). Changes in the intellectual structure of strategic management research: a bibliometric study of the Strategic Management Journal, 1980- 2000. Strategic Management Journal, 25, 981-1004.

SEBRAE (2009). Estudo de Tendência: Perspectivas para a Panificação e Confeitaria 2009-2017. Disponível em: http://www.aipesp.org.br/caderno de tendencias.pdf

Vergara, S. C. (2010). Métodos de pesquisa em administração. São Paulo: Atlas. 